\title{
Corona Virus Health Crisis (COVID-19) and Reconfiguration of Stability/Power Poles in the International System
}

\author{
Abdoulaye MFONKA 8 (D) $\square$ \\ Chargé de cours, Département de science politique, FSJP, Université de Yaoundé II-Soa \\ $\triangle$ Corresponding Author: Abdoulaye MFONKA, E-mail: abdoumfonka@gmail.com
}

\section{ARTICLE INFORMATION}

Received: February 08, 2021

Accepted: March 05, 2021

Volume: 3

Issue: 3

DOI: $10.32996 /$ jhsss.2021.3.3.5

\section{KEYWORDS}

Corona virus; reconfiguration;

stability pole; international system

\section{ABSTRACT}

The international system is regularly changing whether in times of peace or crisis. This sometimes makes it very complex to determine precisely who the "policeman of the world" is. This position varies according to the context. Since the end of 2019, the world has been facing a severe health crisis caused by a new virus, SAR-Cov 2, also known as Coronavirus or Covid-19. The question on everyone's minds is whether this health crisis will redefine of the poles of stability and power in the global system. It appears to be a strong factor that will revive the latent and manifest positioning struggles that have shaken the international system since the end of the cold war, a system seized by unimultipolarity and the leadership quarrels of the emerging powers. We may or not end up with the overthrow of the current world order with regards to the managerial approach to the crisis by the various world powers.

\section{Introduction}

L'avènement de la COVID-19 qui frappe le monde entier depuis quelques mois sera à l'origine de l'apparition d'un nouveau paradigme. Avant toute analyse, il est nécessaire de rappeler que le nom de la maladie est Corona Virus Disease (SRASCoronavirus 2) très souvent appelé Covid-19. C'est un nouveau virus de la famille des ribovirus (virus à ARN) appelés " corona » parce que leurs protéines d'attache ressemblent à des pics, ce qui en coupe donne un aspect rappelant une couronne ${ }^{2}$. II est officiellement apparu en Chine dans la vallée de Wuhan le 31 décembre 2019, date à laquelle a été signalé un nouveau virus causant une nouvelle variété de syndrome respiratoire aigu.

Nous connaissons la formule de Giuseppe Tomasi di Lampedusa dans Le Guépard, «pour que tout reste comme avant, il faut que tout change ${ }^{3}$ » (Giuseppe, 2006). Ainsi, "À chaque crise politique et sociale se développent des pratiques d'autonomie et d'autoorganisation dont les contenus et les formes sont suscités par les rapports sociaux capitalistes en pleine transformation; contenus et formes qui peuvent dans un second temps, et en général très vite, entrer en opposition avec la logique capitaliste qui a provoqué leur apparition, et devenir alors des points d'appui pour une lutte anticapitaliste »(Collonges, 2015). Si un autre monde est possible, et il l'est, c'est souvent dans les moments de crise majeure qu'il émerge au grand jour. A titre d'illustration, la seconde guerre mondiale en 1945 où le monde était dominé par deux grandes puissances : d'un côté les Etats-Unis d'Amérique et leurs alliés (Bloc de l'Ouest); et de l'autre l'Union soviétique et ses alliés (Bloc de l'Est) et on parlait alors d'un monde bipolaire. II est également possible d'évoquer la chute du Mur de Berlin, l'effondrement de I'Union soviétique en 1989 ainsi que la fin de la guerre froide, qui a conduit à un monde unipolaire avec la superpuissance des Etats-Unis d'Amérique.

Néanmoins, plus tard, plusieurs facteurs vont atténuer la toute-puissance américaine sur la scène mondiale notamment la résurrection de la Russie sur le plan stratégico-militaire, la puissance économique de l'Union européenne, la montée en puissance de la Chine sur le plan économique ainsi que la montée du terrorisme international qui connaitra son essor avec l'attentat du 11 septembre 2001 d'où la configuration uni-multipolaire de la société internationale.

\section{K C AL-KINDI CENTER \\ $\mathbf{R}$ D FOR RESEARCH AND $\mathbf{R}$ D DEVELOPMENT} Your gateway to world-class research

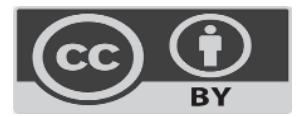

Published by Al-Kindi Center for Research and Development. Copyright (c) the author(s). This open access article is distributed under a Creative Commons Attribution (CC-BY) 4.0 license

\footnotetext{
${ }^{2}$ Gérard Chaouat est médecin, immunologiste et chercheur au CNRS en France.

${ }^{3}$ On peut voir ici la séquence du film éponyme de Luchino Visconti sur www.allocine.
} 
C'est peut-être aussi le cas aujourd'hui avec la crise sanitaire mondiale actuelle(COVID-19) qui va certainement reconfigurer l'ordre international établi en ouvrant une fenêtre d'opportunité aux acteurs/puissances émergents comme la Chine, autrefois considérée comme "une menace » afin de faire valoir les bénéfices politiques de son modèle autoritaire et surtout mettre en évidence les carences d'un système dans son ensemble, celui du multilatéralisme hérité de 1945 et sa machinerie institutionnelle aujourd'hui grippée. C'est dire que les signes avant-coureurs d'un bouleversement avenir de l'ordre international étaient déjà perceptibles avant la propagation du coronavirus. On doit donc y voir l'émergence d'un nouveau paradigme dans les relations internationales, structurant la société internationale. C'est alors que le choc mondial actuel se transformera non seulement en crise politique mais aussi en crise économique d'autant plus profonde (peut-être comparable dans son ampleur à celle de 1929) que l'économie mondiale n'était pas vraiment sortie de la crise de 2008. Dès lors, comment comprendre que la crise de la Covid19 constitue un déterminant clé de rupture d'un ordre mondial balbutiant depuis plusieurs années (1) et quelle seront les conséquences de cette crise sur la configuration de la scène internationale aujourd'hui(2).

\section{La crise sanitaire Covid-19 comme catalyseur/déterminant de la mutation latente et progressive d'un système international balbutiant}

Le monde a toujours été en pleine ébullition depuis la nuit des temps, partagé entre les moments de stabilité et d'instabilité ; les querelles de leadership entre les puissances mondiales et les crises diverses. Plusieurs facteurs sont généralement à l'origine de ces mutations et parfois même en temps dit de paix, nous vivons des conflits latents entre les puissances stabilisatrices ou " gendarmes » du monde. La crise de la Covid-19 vient comme un déterminant fort qui va raviver les luttes de positionnement latentes qui secouent le système mondial depuis quelques années. Dès lors, on se demande si la pandémie viendra mettre fin au monde existant pour en créer un nouveau ou alors s'il n'y pas de véritables changements.

\subsection{La guerre politico-économique et les querelles de leadership entre les puissances stabilisatrices de la scène internationale avant la Covid-19}

La structure du système international fait allusion aux rapports de force entre puissances et à la distribution de la puissance. Cette structure variera donc en fonction du nombre de grandes puissances agissant sur la scène internationale et des changements dans la distribution des capacités de puissance entre les unités du système. Evolutive, la structure se forme par la coexistence des unités du système international, leur coaction et les résultats de ces dernières. Elle définit donc la configuration des différents éléments du système, c'est à dire l'ordre mondial, toujours provisoire. Et si pour la plupart des réalistes, l'économie n'est pas essentielle en soi $^{4}$ (Gilpin, 1981), elle l'est toutefois indirectement dans la mesure où la puissance militaire d'un État dépend en grande partie de ses moyens économiques. II n'est donc pas à exclure que cette crise, qui touche presque tous les pays, mais de manière inégale ${ }^{5}$ ait des répercussions sur l'équilibre de la puissance.

Dans I'histoire des relations internationales, si la chute du mur de Berlin, en 1989, avait fait naître l'espoir d'un monde plus coopératif guidé par des normes internationales bien définies, les attentats du 11 septembre 2001 ont plutôt souligné de manière brutale le retour de la realpolitik et ont remis à l'avant-plan la poursuite et la protection des intérêts nationaux des Etats. Alors que la guerre froide offrait une vision manichéenne des relations entre les États et de la façon dont ceux-ci déterminaient leurs objectifs et leurs ambitions sur la scène internationale, les bouleversements du monde à l'heure de la montée en puissance de la Chine, des provocations de l'Iran ainsi que de la Corée du Nord et des tensions épisodiques entre la Russie et les États-Unis nous démontrent que les enjeux sont dorénavant beaucoup plus complexes.

Mongrenier pense à son tour que, si le «monde unipolaire» de l'après-Guerre froide a été abondamment vilipendé, l'expression n'était que flatus vocis ("souffle de voix») (Mongrenier, 2014). L'unification de la planète par la finance, le commerce et les technologies de l'information n'impliquait pas un pouvoir centralisé entre les mains d'un détenteur unique de la puissance. Aussi le «moment unipolaire» s'est-il révélé bref, l'expression ne traduisant que très imparfaitement la situation internationale de l'époque ${ }^{6}$. En quelques années, le thème de la multipolarité s'est imposé, comme programme et anticipation de l'avenir, puis comme une pseudo-évidence. Cette représentation globale est sous-tendue par l'émergence de nouvelles puissances économiques, anciens tributaires et colonisés situés à la périphérie du système occidental. De fait, l'extension des logiques de marché à l'Est et au Sud ont fait entrer des pays non-occidentaux dans le jeu économique global.

\footnotetext{
${ }^{4}$ La plus belle exception quant au caractère secondaire de l'économie chez les réalistes se trouve chez Robert Gilpin dans son fameux ouvrage War and Change in World Politics.

${ }^{5}$ Le modèle de croissance économique américain, reposant sur une relative faiblesse de l'État providence et un marché du travail flexible le rend particulièrement vulnérable aux chocs exogènes, surtout quand ceux-là génèrent du chômage. Pour plus de details, voir Mark Blyth, « The U.S. Economy Is Uniquely Vulnerable to the Coronavirus», Foreign Affairs, 30 mars 2020.

${ }^{6}$ L'expression de «moment unipolaire» rend mieux compte de l'état d'esprit des dirigeants américains que celle de «monde unipolaire», expression qu'ils n'ont d'ailleurs guère reprise à leur compte. Au faîte de leur puissance, les États-Unis approchent des limites de leur potentiel et leurs dirigeants anticipent la modification à venir des équilibres mondiaux. Dès lors, il s'agit d'utiliser au mieux une fenêtre d'opportunité de quelques années pour «mettre en forme» le monde et préparer l'insertion des puissances ascendantes dans un système international dont les principes et les structures auront été largement définis par les Occidentaux. II faut donc se mouvoir, frapper si nécessaire, anticiper et poser des règles. Cet état d'esprit constitue la toile de fond des engagements militaires américano-occidentaux en Afrique du Nord, au Moyen-Orient ou en Haute-Asie (Libye, Irak, Afghanistan), ces différents théâtres relevant du «Grand Moyen-Orient».
} 
Après les NPIA ${ }^{7}$, c'est le tour de la République populaire de Chine, de I'Inde, du Brésil ou encore de la Russie, celle-ci représentant un cas spécifique. Précédemment élaborée pour désigner de nouvelles opportunités financières, l'expression de «marché émergent» laisse place à celle de "puissance émergente ${ }^{8}$ ». En 2001, Jimmy O' Neil, chef économiste à la Goldman Sachs, forge le concept de «BRIC». II s'agit de pointer les dynamiques de croissance et le potentiel du Brésil, de la Russie, de l'Inde et de la Chine (Mongrenier, 2014).

Si les Etats-Unis se trouvent depuis la fin de la guerre froide dans une position semblable que celle de la Grande Bretagne après la bataille de Waterloo à savoir une position prédominante, les autres puissances se sentent menacées par la puissance hégémonique, que représente Washington. La prépondérance ayant de surcroît un coût important, tôt ou tard, il y a un déclin relatif, alors que parallèlement les autres puissances montent en puissance. Washington verra donc dans un avenir certain sa puissance être d'abord rattrapée, ensuite égalée et enfin peut-être dépassée ( Smouts, Battistella, et Venesson, 2003).

Afin de rééquilibrer le système, les puissances émergentes : Russie, Inde, Japon, Chine, Union européenne, Brésil sont en effet de plus en plus tentées de se retrouver en position de porte-à-faux vis-à-vis des Etats-Unis. Leur objectif est de créer un monde multipolaire qui affaiblirait du même coup la puissance américaine, laquelle, selon de nombreux chercheurs ne constitue en conséquence qu'un «intermède stratégique», bientôt dépassé. Aussi la question n'est-elle pas de savoir si d'autres puissances chercheront à rétablir un équilibre, mais plutôt quand elles y parviendront. L'axe franco-allemand par le biais de I'Union européenne, la Chine du fait de sa récente croissance (l'adoption de capitalisme aidant), l'Inde, le Japon et la Russie, tous revendiquent sans doute légitimement leur part dans la paternité de la nouvelle donne. Il existe donc une réelle volonté de rééquilibrer le système international ${ }^{9}$.

C'est ainsi que "Washington fait face au classique défi, en relations internationales, de répondre à une puissance émergente dans une région où les États-Unis ont été longtemps prédominants, y possédant de substantiels intérêts économiques et des accords de défense (cinq des sept traités de défense mutuelle signés par les États-Unis concernent l'Asie). Les responsables politiques se sont sentis de plus en plus concernés par l'impact relatif de la montée de la Chine dans la région et par la possibilité de voir l'émergence chinoise y précipiter l'instabilité, sous la forme d'un conflit armé à propos de Taiwan, ou d'autres disputes intra régionales ${ }^{10}$ ".

Le système international est alors déstabilisé et à la recherche d'un nouvel équilibre, du fait de la montée inexorable d'une autre puissance majeure, corrélative du déclin de la puissance dominante du moment. Admettre qu'une telle transition, avec les dangers qu'elle recèle, est aujourd'hui engagée entre la Chine et les États-Unis, justifie la stratégie proposée par Ikenberry lorsqu'il affirme que: "Le "moment unipolaire" américain doit inévitablement prendre fin. Si la lutte décisive du vingt-et-unième siècle est entre la Chine et les États-Unis, la Chine devrait avoir l'avantage. Si la lutte décisive est entre la Chine et le système occidental revivifié, l'Occident doit triompher » (Ikenberry, 2009). Dès lors, quelle pourra être la contribution de la Covid-19 à cette dynamique de la géopolitique mondiale.

\subsection{L'avènement de la pandémie de la Covid-19 comme catalyseur et accélérateur d'une mutation géopolitique mondiale}

La crise actuelle coïnciderait avec le passage délicat d'un monde ancien, qui tarderait à céder la place, vers un monde nouveau et incertain qui peine à s'affirmer et à lui succéder. Les géographes ont souligné la récurrence des crises économiques: "Leur fréquence s'est accrue avec l'industrialisation et l'essor des échanges internationaux, et leur nature a également évoluée. Aujourd'hui, l'intégration des économies productives et des marchés financiers favorise la diffusion planétaire de ces crises (...) » (Ghorra-gobin, 2012). Qu'en est-il de la crise sanitaire de la Covid-19?

II est clair que la Covid-19, n'est ni la variole, ni l'Ebola, encore moins le VIH et que son actualité n'est pas vécue de la même façon entre I'hémisphère nord, obsédé par le "zéro mort" permanent et l'hémisphère sud habitué à des désastres épidémiques ${ }^{11}$ voire à des destructions de masse (3 millions d'enfants de moins de 5 ans meurent tous les ans de malnutrition et 25000 personnes meurent chaque jour de faim dans le monde... la guerre civile au Rwanda a fait entre 500000 et 1 million de morts...) $)^{12}$.

\footnotetext{
${ }^{7}$ NPIA : Nouveaux Pays Industriels d'Asie.

${ }^{8}$ Le concept de «marché émergent » est utilisé par Antoine van Agtmael (Bankers Trust, New-York) dès 1981 pour désigner les NPIA (Nouveaux pays industrialisés d'Asie-Pacifique) de l'époque. Sur la généalogie de cette représentation globale de nature économique, financière et géopolitique, voir Michel Foucher, "Les nouveaux (dés)équilibres mondiaux », Documentation photographique, dossier n 8072, novembre-décembre 2009, pp. 2-6.

${ }^{9}$ Pour plus de details lire David Skidmore, «Understanding the Unilateralist Turn in U.S. Foreign Policy», in Foreign Policy Analysis, 2005, 2, pp.207-228.

${ }^{8}$ Zitoellick et Dai Bingguo, alors vice-ministre des Affaires étrangères chinoises lors du «Senior dialogue», P 147.

${ }^{11} \mathrm{Cf}$ l'étude faite par MSF sur l'Ebola, Marburg et les fièvres hémorragiques sur le continent africain https://www.msf.fr/eclairages/ebola-marburg-et-les-fievresconsulté le 22 juin 2020.

12 Ibid.
} 
Globalement, les crises sanitaires sont des crises politiques, économiques et sociales, parce qu'elles ont des conséquences politiques, économiques et sociales, mais également parce qu'elles ont des causes politiques, économiques et sociales. Les crises peuvent aussi révéler des faiblesses structurelles des sociétés. Aujourd'hui, avec la Covid-19, les difficultés qu'ont les hôpitaux à gérer la pandémie dans un certain nombre de pays européens sont clairement la conséquence du désinvestissement public dans ce secteur. Elles ont également mis au jour la forte dépendance de l'Europe vis-à-vis de l'étranger, notamment de la Chine et de l'Inde, pour un certain nombre de produits stratégiques et vitaux dans cette crise, comme les masques de protection, les respirateurs artificiels ou les médicaments.

C'est ainsi qu'en l'espace de quelques mois, le coronavirus a chamboulé l'orthodoxie économique mondiale, indissociable du Consensus de Washington ${ }^{13}$. La crise de la COVID-19 met en lumière l'effritement de la domination du discours néolibéral en matière de développement et renforce ainsi l'antagonisme entre deux modèles, qui soutient la rhétorique d'une " nouvelle guerre froide » entre la Chine et les États-Unis sans oublier le jeu trouble de l'Europe. La pandémie avive des tensions latentes, potentiellement explosives. C'est un court-circuit durable de la mondialisation, qui s'inscrit dans des cycles, déjà enclenchés, de coopération, de compétition et de confrontation cognitive, c'est-à-dire de mobilisation, d'orientation et de contrôle des cerveaux.

D'emblée, la crise aura des conséquences nombreuses sur la vie sociale, politique et économique. Par exemple, les impacts de la Covid-19 sur l'offre et la demande alimentaires affecteront directement et indirectement les piliers de la sécurité alimentaire et de la nutrition (SAN):disponibilité, accès, utilisation et stabilité. On s'attend également à ce que les mesures de confinement adoptées dans plusieurs pays aient des effets immédiats, et ces mesures auront également des répercussions à plus long terme sur l'ensemble de l'économie mondiale. La pandémie de la Covid-19 affecte même déjà les systèmes alimentaires directement par ses effets sur l'offre et la demande de nourriture, et indirectement par la diminution du pouvoir d'achat, de la capacité de production et de distribution de nourriture, et l'intensification des tâches de soins, qui auront tous des impacts différenciés et affecteront plus fortement les pauvres et les personnes vulnérables.

La propagation du virus Covid-19 n'aura pas uniquement des conséquences sanitaires et humaines. Elle aura aussi des impacts forts sur les activités économiques. En effet, selon les prévisions de l'OCDE le 2 mars 2020, la croissance économique mondiale serait de 2,4\% soit une baisse de 0,5\% par rapport à la prévision initiale de croissance déjà faible de 2,9\% de novembre $2019{ }^{14}$ (OECD, 2020). La Covid-19 a aussi des incidences profondes sur les performances des marchés du travail. Au-delà de la question urgente de la santé des travailleurs et de leurs familles, le virus et les chocs économiques qui en découlent ont des répercussions de trois ordres sur le monde du travail: la quantité d'emplois (à la fois le chômage et le sous-emploi); la qualité du travail (par exemple, salaires et accès à la protection sociale); et les effets sur des groupes particuliers qui sont plus vulnérables face aux mauvaises performances du marché du travail.

Les crises économiques, commerciales, budgétaires ou encore financières découlant de la pandémie sont telles qu'elles ont converti au keynésianisme bien des pays qui y étaient réticents. Mais cette conversion en masse n'est que la révélation de mouvements antérieurs qui s'affichent à la faveur de la crise. Ainsi les débats budgétaires en Europe ont changé de taille mais pas de nature : le saut est quantitatif, pas (encore) qualitatif vers une plus ou moins grande intégration.

Alors que le contraste est grandissant entre I'Asie orientale et le reste du monde dans la gestion et le traitement de la Covid-19, tout particulièrement le monde occidental. En tardant souvent à tirer les enseignements du traitement de la pandémie par les pays d'Asie de l'Est, ne pouvant s'appuyer sur une base industrielle et nationale aussi solide que chez les puissances asiatiques, en se divisant entre principe de confinement de très grande ampleur (Italie) et logique de dépistage généralisé (Allemagne), en ayant un nombre de victimes déjà largement supérieur à la Chine (même si les accusations de sous-estimation par les autorités du nombre de morts sont légion) pour une population totale nettement moindre, l'Europe et les États-Unis (où certaines estimations font état de 2000000 de victimes potentielles) paraissent confirmer la perte de "leur monopole de puissance » (Védrine, 2017) au sein d'une planète dans laquelle la Covid-19 pourrait accélérer le "requiem pour le monde occidental » (Boniface, 2019).

En outre, on constate que le Conseil de sécurité des Nations Unies n'a pas pu s'accorder sur une résolution sur la Covid-19 faute d'un accord entre les États-Unis et la Chine. C'est une réalité inédite, puisque même durant la guerre froide les États-Unis et I'URSS étaient parvenus à se mettre d'accord pour favoriser la recherche d'un vaccin contre la polio. Le G7 n'est pas plus parvenu à se mettre d'accord sur un texte, un État voulant qualifier la Covid-19 de «virus chinois ». On assiste à un véritable blame game entre les États-Unis et la Chine, qui éclaire en réalité un déficit de leadership mondial.

\footnotetext{
${ }^{13}$ Le terme de Consensus de Washington a été inventé en 1989 par un économiste américain, John Williamson. L'expression renvoie à dix prescriptions économiques recommandées par les institutions de Bretton Woods aux pays en émergence, notamment en Amérique latine, ayant fait appel à elles dans les années 1980-1990.

${ }^{14}$ OECD "Coronavirus: the world economy at risk", OECD interim Economic Assessment, 2 march 2020.
} 
Au-delà de tout ceci, il faut comprendre que les crises sont aussi des opportunités politiques, économiques et sociales. Elles peuvent présenter une opportunité pour changer la société, en permettant de prendre des décisions que l'on n'aurait pas pu prendre dans d'autres circonstances. La Covid-19 pourra donc servir de prétexte comme de catalyseur vu les agendas en cours en termes de rapports de force entre les grands protagonistes des équilibres mondiaux. Le choc du Coronavirus ne sera pas seulement sanitaire mais aussi économique et géostratégique.

Il constitue donc un test de la résilience des systèmes politico-économiques dans un contexte exacerbé par la rivalité sinoaméricaine en même temps qu'elle souligne la fragilité de l'Union européenne. L'avènement de la pandémie sera considéré comme un des faits majeurs déclencheur et accélérateur des querelles de leadership entre les grandes puissances mondiales. Pour ainsi dire, la Covid-19 sera comme «la goutte d'eau qui fait déborder le vase »15.

\section{Les conséquences de l'après crise sanitaire : entre positionnement de la Chine et reconfiguration des pôles de stabilité/puissance dans le système international}

La pandémie de la Covid-19 qui s'est répandue dans toutes les sociétés aura des conséquences immenses sur les relations entre les États, leurs fonctionnements et l'équilibre des puissances. De manière invisible, se joue une profonde redistribution du pouvoir non seulement entre les États-Unis, la Chine et I'UE mais aussi entre les États nationaux et les grandes plates-formes numériques. Qui des premiers ou des secondes sont aujourd'hui, seront demain, les principaux organisateurs de nos vies?

La République populaire de Chine pourra profiter du bouleversement du système international pour se positionner sur l'échiquier international. Et quelle pourra être la nouvelle configuration de la géopolitique mondiale ?

\subsection{Le positionnement de la chine dans le nouvel ordre mondial après la crise sanitaire de la Covid-19}

Bien avant le déclenchement de l'alerte pandémique l'état des lieux géopolitique était relativement simple : tous les grands protagonistes qui maitrisent l'essentiel des mécanismes de la mondialisation (Chine, Etats-Unis, Europe) étaient en équilibre instable et cherchaient à retrouver des appuis en interne pour garantir et affirmer leurs manœuvres externes en termes d'affirmation de leurs leaderships. C'était le cas de la Chine, épicentre de cette catastrophe sanitaire mondiale, qui mettait tout en œuvre pour tenter de dépasser et déclasser les Etats-Unis en termes de puissance autour de 2021, année symbolique pour Xi Jinping avec le centenaire du Parti Communiste Chinois.

A cet effet, la République populaire de Chine pourrait sortir finalement grandie de sa gestion de crise alors qu'elle paraissait, à priori, fragilisée par la crise de la Covid-19, accusée de l'avoir sous-estimée et d'avoir tenté d'en nier l'ampleur. De plus, certains observateurs considéraient que cette pandémie serait pour elle ce que Tchernobyl fut pour I'URSS.

"La Chine pourrait vraisemblablement bien devenir la plus formidable superpuissance dans la compétition globale avec les États-Unis » (Mearsheimer, 2001). "On le dit et le répète. Devenue une puissance montante, la Chine serait seule capable de rivaliser avec les Etats-Unis à l'horizon 2025. Dans cinquante ans au plus tard, selon certains experts, le pays pourrait même dépasser l'ensemble de l'Europe ${ }^{16}$ ». L'adhésion de la Chine à l'Organisation mondiale du commerce (OMC) en 2001 a consolidé l'intégration du pays dans l'économie capitaliste internationale au point de devenir un acteur incontournable du système économique et politique international. Au niveau régional, elle devient progressivement le pôle structurant d'un réseau d'échanges régional au point de susciter des interrogations sur un éventuel recentrage de l'économie planétaire autour de l'Asie.

La Chine proposerait, en fait, une voie plus prometteuse. Elle serait en train de faire la preuve qu'un régime autoritaire est mieux à même d'assurer la prospérité qu'un régime démocratique. Inévitablement, elle aurait la capacité, non seulement par le "soft power », mais surtout par l'exercice de sa puissance économique, et à l'avenir de sa puissance militaire (le « hard power ») qui ne peut qu'immanquablement en résulter, d'imposer au monde sa vision, de faire régner sa loi.

La Chine a su profiter de la mondialisation pour rattraper une partie de son retard et s'approprier les connaissances technologiques avancées d'autres pays (Lewis, 2019). Plusieurs méthodes ont été employées : les Chinois ont pris des parts dans des entreprises étrangères pour accéder aux technologies récentes, ils ont imposé l'obtention de savoir-faire de pointe lors de discussions pour des accords commerciaux, ils ont envoyé des étudiants dans des universités étrangères pour observer l'état de la recherche scientifique.

Pékin a eu, par ailleurs largement recours au cyber espionnage pour piller des données ou copier des projets technologiques ambitieux comme l'avion F-35, à tel point qu'un accord entre les présidents Obama et Xi fut signé en 2015 pour lutter contre le piratage informatique. Elle s'est aussi investie dans le domaine du sport.

\footnotetext{
${ }^{15}$ Désigne un ras le bol, une situation qui a dépassé les bornes. L'expression fait bien le parallèle entre la tension ou l'énervement qui monte (le vase qui se remplit jusqu'à être bien plein) et l'explosion qui suit (le vase qui finit par déborder dès que la petite goutte de trop y a été versée).

${ }^{16}$ Lire le dossier «Chine» du Monde diplomatique n607, Octobre 2003, p. 14-19; et L'Atlas du Monde diplomatique, Le Monde diplomatique, Paris, janvier 2003, p. 156-159.
} 
C'est en cela que la tenue des Jeux Olympiques de Beijing, du 8 au 24 août 2008, exacerbe l'expression internationale de sentiments ambivalents à l'égard de la Chine. Les Jeux représentent incontestablement une manifestation majeure de « soft power ». En cela, ils constituent un enjeu considérable pour les autorités chinoises. Ils seront d'ailleurs perçus à travers le monde comme une consécration de la puissance chinoise.

En outre, la présence chinoise est également très forte dans les pays en voie de développement, où elle construit des infrastructures, mais investit surtout dans l'exploitation des ressources naturelles. Principal économiste de la banque HSBC, Stephen King le dit: "Le monde où nous vivons n'est plus dirigé par les États-Unis mais par les marchés en expansion, dont le moteur, cela va de soi, est la Chine » (King, 2010). La Chine est donc le principal facteur du bouleversement des flux commerciaux dans le monde.

Aujourd'hui, face à la crise sanitaire de la Covid-19, la Chine montre sa capacité à répondre à l'urgence sanitaire par l'organisation d'une véritable médecine de guerre sur le théâtre même d'opérations.

La construction, en un temps record, d'hôpitaux de campagne, la livraison massive de masques, le dépistage systématique de la population, ont frappé les esprits en termes de capacité du système de santé national chinois à répondre avec célérité à l'ampleur de l'urgence. De plus, la capacité du pouvoir en place à démontrer, à la population chinoise et au monde plus largement, son aptitude à relever des défis d'envergure planétaire. La Chine cherche à souligner que sa volonté d'être la première puissance mondiale n'est pas qu'un vœu illusoire. Passée économiquement et socialement du stade de quasi-pays moins avancé (PMA) à la fin des années 1970, à celui de puissance émergente avec les années 1990, pour apparaitre aujourd'hui comme le "numéro bis » (Védrine, 2017) de la superpuissance états-unienne, la Chine incarne un modèle géopolitique à part entière. Le " rêve chinois » repose moins sur la séduction en termes de valeurs ou de fonctionnement politique que sur la performance géoéconomique et sociale et sa quête de domination internationale.

Or, il est frappant de constater qu'une Chine qui a su gérer la crise de la Covid-19, face à une Europe et des États-Unis gravement affectés par la pandémie, s'impose en force dans l'ordre géopolitique international.

Nous assistons de ce point de vue à un spectacle sans précédent: la République populaire de chine d'abord en difficulté au début de la crise, du fait de son attitude initiale de répression des "lanceurs d'alertes" de Wuhan ; obligée de fermer ses usines ; puis paraissant vaincre la pandémie grâce à des mesures autoritaires de quarantaine, combinées avec un usage sans précédent de l'intelligence artificielle; s'en sort enfin de l'épreuve alors que les Européens, devenus le principal foyer d'infection, tardent à mettre en œuvre des mesures drastiques. De même que l'administration Trump qui fait la démonstration de son incompétence brouillonne. La Chine aujourd'hui relance son économie alors que les bourses s'effondrent en Occident, elle contre les insinuations xénophobes déplacées de Donald Trump dans un combat de désinformation absurde, et surtout elle fait figure de planche de salut pour l'Italie ou la Serbie, en partie du fait des maladresses de leurs partenaires européens, avant certainement d'apparaître dans le monde émergent dans le rôle de puissance secourable qui était jadis le rôle naturel des États-Unis.

Dans cette perspective, la pandémie actuelle marquerait la consécration de lignes de forces que l'on a vu se mettre en place ces dernières années, elle pourrait même constituer un "accélérateur"17. Les Occidentaux surmontent beaucoup plus difficilement que l'Asie les difficultés de la crise sanitaire. Le flambeau de l'initiative et du leadership passerait à la Chine, d'abord dans le domaine des "enjeux globaux" (dont la santé fait partie, comme le développement ou le changement climatique), mais aussi bien sûr sur le plan économique (l' "atterrissage économique" dans l'après Covid-19 sera aussi important que la victoire contre la pandémie), technologique (5G), voire un jour militaire (Duclos, 2020).

\subsection{Vers une nouvelle configuration des pôles de stabilité/puissance et une restructuration de la scène internationale après la Covid-19?}

S'il est vrai que nous vivons dans un monde avec trois grandes puissances, les États-Unis, l'Europe et la Chine. La gestion de la guerre contre la Covid-19 aura-t-elle un impact sur la restructuration de l'ordre mondial ?

II est à noter que le monde d'après la crise COVID-19 est en pleine gestation et mutation. En effet, la catastrophe sanitaire et la récession économique ont d'ores et déjà accentué et modifié les rapports de force internationaux. Comme toutes les crises, celle que traverse le monde a non seulement avivé les rivalités latentes, révélé des transformations jusqu'ici occultées et créé de véritables ruptures.

Loin de consacrer un changement de paradigme, la crise sanitaire pourrait accentuer d'abord la rivalité sino-américaine. Ensuite, I'impuissance, la division et la vulnérabilité de I'UE. Tout comme les États-Unis, le Vieux Continent a largement sous-estimé l'ampleur de la pandémie et a tardé à prendre des mesures appropriées, au point où la situation sanitaire s'est très fortement dégradée, particulièrement en Italie et en Espagne, ainsi que l'évolution de la gouvernance mondiale, le recul du

17 Selon l'expression de Dominique Moisi 
multilatéralisme; alors que cette crise est par nature globale et requiert par conséquent une approche coordonnée et coopérative, la prédominance des actions unilatérales étant frappante.

De nombreux aspects liés à cette crise, notamment les approvisionnements en équipements médicaux et en médicaments, alimentent la rhétorique de l'administration Trump ( Rachman, 2020), en faveur d'un découplage des chaînes de valeur, afin de ne pas se placer sous la dépendance de la Chine. Le monde d'après la crise sera bien plus brutal car les plus puissants d'avant la crise risquent bien d'être encore plus hégémoniques qu'avant la crise.

Pendant cette crise, si la " guerre commerciale » qui oppose les Etats-Unis et la Chine constitue la facette la plus visible de la rivalité sino-américaine, derrière les sanctions et contre-sanctions commerciales, c'est en réalité la course à la suprématie mondiale qui se joue. II faudrait que I'UE soit autrement plus forte qu'elle ne l'est aujourd'hui pour être en mesure de tirer son épingle du jeu.

On peut penser que la pandémie va enfin permettre à la Chine, même si aux yeux de certains, ce pays porte une lourde responsabilité dans la propagation de la pandémie, de s'affirmer comme une nation disposant d'un savoir-faire qui a rendu possible la circonscription de la pandémie et de se présenter comme une puissance avec laquelle il va falloir compter dans les prochaines années. Alors, irons-nous vers une valorisation et une reconfiguration du système multilatéral, dans un sens de l'inclusivité, de la solidarité et de l'équité ou, céderons-nous aux sirènes du repli nationaliste qui ne fera qu'exacerber les fractures entre les nations?

La pandémie de la Covid-19 est donc une crise sanitaire et économique majeure qui remet en cause les principes même de mondialisation et de globalisation. Cette pandémie ne marquera certes pas la fin de la globalisation. Mais elle remettra en cause un certain nombre de ses modalités, et de ses présupposés idéologiques, dont notamment le fameux triptyque néolibéral : ouverture des marchés, recul de l'État, et privatisations. Si cette remise en cause était déjà engagée avant même le début de la crise, elle s'accentuera par la suite. Aussi, il faudra que nos systèmes économiques mondiaux et interdépendants soient plus adaptés à ce type de crise. Ainsi la crise de la Covid-19 va mettre en évidence le fait que la globalisation accroît la vulnérabilité des nations qui ne prennent pas les précautions nécessaires pour assurer leur sécurité au sens large.

En outre, son impact sur la globalisation sera plus sur la remise en cause d'une certaine uniformisation des modèles mondiaux. En cela cette pandémie aura sûrement des effets induits sur les modes de représentation que nous pouvons avoir du réel en réintroduisant à la marge des approches locales et culturelles dans les modes de productions et de consommation.

Le fait que, pour la première fois depuis la création des Nations unies, une pandémie n'entraîne pas de consensus constitue un bien mauvais présage. Cette situation résulte à la fois des désaccords entre États et du désintérêt de certains d'entre eux pour tout leadership international. Tout ceci est extrêmement préoccupant, car on sait bien qu'une coordination internationale forte peut faire la différence.

La crise sanitaire de la Covid-19 constituera également une épreuve politique pour les systèmes démocratiques européens. Comme toujours, ce sont les crises qui révèlent aux sociétés leurs forces et leurs faiblesses. D'ores et déjà se mettent en place des narratifs politiques pour préparer l'avenir. Trois narratifs sont en concurrence : le populiste, l'autoritaire qui le rejoint sur bien des points, et le démocratique.

C'est désormais une épreuve majeure à laquelle est confronté l'ensemble de la communauté internationale. La crise affecte d'abord les systèmes de santé, les économies, mais aussi la gouvernance des pays dont la population est contaminée, à travers la capacité de leurs autorités à évaluer la situation épidémiologique et à adopter des mesures de protection idoines. Sur le plan global, cette pandémie illustre le niveau d'interdépendance des économies globalisées et la vulnérabilité des chaînes de valeur en cas de perturbation majeure.

\section{Conclusion}

La crise du coronavirus agit comme un catalyseur de la rivalité entre les puissances mondiales. L'ampleur de la récession économique qui s'annonce et la façon dont les économies américaines et chinoises sauront la maîtriser, en définiront le ton et le tempo sans oublier le rôle de l'Europe dans ce jeu. La pandémie actuelle marquerait la consécration de lignes de forces que l'on a vu se mettre en place ces dernières années. De façon générale, se jouera une profonde redistribution du pouvoir entre les États-Unis, la Chine et l'UE. Elle pourrait enfin, permettre à la Chine de réaliser son « rêve » de superpuissance mondiale face à I'hégémonie américaine. Globalement, on dirait que dans la géopolitique mondiale, la crise va exacerber les grandes tendances déjà à l'œuvre sans changer fondamentalement la donne. La crise du coronavirus pourrait accélérer la fin d'un monde et la naissance d'un nouveau. 


\section{References}

[1] Cynthia Ghorra-gobin, (dir.), (2012), Dictionnaire critique de la mondialisation, Paris, Armand Colin.

[2] David Skidmore (2005), «Understanding the Unilateralist Turn in U.S. Foreign Policy», in Foreign Policy Analysis.

[3] Gideon Rachman (2020), « Nationalism Is a Side Effect of Coronavirus », The Financial Times, 24 mars2020, disponible sur : www.ft.com.

[4] Giuseppe Tomasi di Lampedusa (2006), Le Guépard, Paris, Le Seuil.

[5] Hubert Védrine (2017), Le monde au défi, Paris, Hachette Pluriel.

[6] James A. Lewis (2019), "Competiting Over Leadership: China vs. the US » in A. Amighini (dir.), China's Race to Global Technology Leadership, Institut pour les Etudes de Politique Internationale.

[7] Jean-Sylvestre Mongrenier (2014), «Feu la multipolarité. L'occident au défi d'un monde chaotique et déséquilibré » in Tribune, Institut Thomas More.

[8] John J. Mearsheimer (2001), The Tragedy of Great Power Politics, New York, Norton \& Company.

[9] John Ikenberry (1999), "Liberal hegemony and the future of American postwar order », in T. V. Paul et John Hall (dir.), International Order and the Future of World Politics (Cambridge: Cambridge University Press, 1999.

[10] John Ikenberry (2009), "The Rise of China and the Future of the West. Can the Liberal System Survive? », Foreign Affairs, 88 (1).

[11] Lucien Collonges (2015), «Demain est déjà commencé », Encyclopédie internationale de l'Autogestion, T. 4, Paris, Syllepse.

[12] Mark Blyth (2020), " The U.S. Economy Is Uniquely Vulnerable to the Coronavirus», Foreign Affairs.

[13] Michel Duclos (2020), "Le Covid-19 est-il un game-changer géopolitique ? », Institut Montaigne, Blog mars.

[14] Michel Foucher (2009), «Les nouveaux (dés)équilibres mondiaux », Documentation photographique, dossier n 8072 , novembre-décembre.

[15] Marcel Merle (1988), Sociologie des relations internationales, Dalloz, $4^{\mathrm{e}}$ éd.

[16] Marie Claude Smouts, Dario Battistella (2003), et Pascal Venesson, Dictionnaire des relations internationales, Paris, Dalloz.

[17] Mario Bettati (1985), Le nouvel ordre économique international, puf, «Que sais-je ? ", $n^{\circ} 2088,2^{\mathrm{e}}$ éd.

[18] Pascal Boniface (2019), Requiem pour le monde occidental. Relever le défi Trump, Paris, Eyrolles.

[19] Raymond Aron (1962), Paix et guerre entre les nations, Calmann-Lévy.

[20] Robert Gilpin (1981), War and Change in World Politics, Cambridge, Cambridge University Press. 